\title{
LoRa based Architecture for Fault Localization in Transmission Lines
}

\author{
Jignesh Gohil \\ Raychem Innovation Center \\ Raychem RPG Private Limited \\ Halol, Gujarat, India
}

\author{
Chintan Parmar \\ Raychem Innovation Center \\ Raychem RPG Private Limited \\ Halol, Gujarat, India
}

\begin{abstract}
In a cloud computing based sensor network, nodes transmit data to the cloud and then data is processed on the cloud. The central processing performed on the cloud consumes precious computing time due to vast amounts of data. In some scenarios, processing can have time constraints. If the data processing can be done using simple algorithms, edge nodes of the sensor network can be used to save transmission and computing time. A concept of fog computing has been introduced here that includes a layer of hierarchy in cloud computing architecture that processes the initial data and quickens the decision-making process. In this research, a fog computing concept is explored for fault localization in transmission lines and distribution networks. In transmission lines and overhead distribution network, the fault detection is very crucial and should take minimum time. When a longrange (LoRa) transceiver based fault indicator is realized for increased range, it presents various challenges to the existing architecture. As the number of data concentrator nodes reduce due to higher range of LoRa, the fault localization complexity increases. In this research, fault indicators are installed with GPS coordinates to reduce complexity for fault localization. Two fault indicators are paired to localize the fault in the transmission lines. To find the location of the fault faster than the time-consuming process of the cloud computing, a new hierarchical layer of fog node is introduced in the system. This new architecture is compared with the earlier system, and the pros and cons are discussed in this research.
\end{abstract}

\section{General Terms}

Fault Localization, LoRa Communication

\section{Keywords}

Fault Indicators, Fog Computing, LoRa Communication, Transmission Line Fault, overhead distribution network.

\section{INTRODUCTION}

Cloud computing offers high computational power and resources for data processing in the cloud but at a higher cost [1]. Along with higher operating costs, cloud also has data security issues that are extensively researched [2]. Numerous solutions have also been offered for these data security woes [3]. Different architecture solutions for cloud computing have been researched to achieve maximum security and minimum time. A hierarchical solution to cloud computing is proposed called as fog computing. In fog computing, the data is computed in a middle layer of hierarchy closer to the ground nodes. Fog computing, also known as edge computing, is a growing trend in the computing domain as cloud computing has issues with security, latency, and high running costs. Adding an extra layer of computing offers better localization of data and better security [4], [5]. As the middle layer computes data from fewer number of nodes with less data, the computing time and power consumption also reduces. Fog computing using the edge nodes has been widely researched in the domain of internet of things (IoT). In [6], the authors use an embedded system for fog computing of a levee monitoring system. Faster response is guaranteed when the computing is done on the edge where the data is generated, even if computing resources do not match that of the cloud. In this research, a hierarchical architecture using fog computing is proposed for fault indication in transmission lines.

Fault Passage Indicators (FPIs) are installed on the transmission lines to indicate faults on the lines. Faults like earth fault, di/dt fault, and phase faults are monitored by FPIs along with current and voltage values of the transmission lines. The FPI monitors the transmission lines in real-time and relays the message of normal operations and faults to main control center. The FPI is a battery powered device which can also draw power from the transmission cables. More number of FPIs gives better view of all the dynamics of the grids but installing them is costly. Numerous algorithms have been researched extensively for optimized placement of fault indicators and retrieve maximum grid data [7].

Progressing from a visual indication of the line fault, FPIs have evolved to become wireless sensor nodes in wireless sensor networks (WSN) to cover remote sites. In typical implementations, the FPIs contain a multi-hop short-range RF transmitter like RF/Zigbee [8] for data transmission. $\mathrm{RF} /$ ZigBee have a very low range of a few meters and thus higher number of data collector gateways are required. This increases the overall costs of the system. Also, the data concentrator communicates in machine to machine (M2M) technique with a Signal Conditioning and Data Acquisition (SCADA) systems that polls the data.

LoRa technology uses $868 \mathrm{MHz} / 915 \mathrm{MHz}$ ISM bands with a coverage of 5 kilometers (3.106 miles) to 15 kilometers (9.302 Miles). The standard implementation of FPIs interface with the data concentrator will change because of this huge range. With the large range, current architecture of RF/ZigBee based FPI will not suffice. Thus, a new architecture with LoRaWAN is proposed with modification to LoRaWAN architecture and current FPI installation architecture with RF/Zigbee.

Rest of the paper is organized as follows. Section II discusses current implementation of FPI using Geographical Information System and cloud. Section III discusses a standard implementation of FPIs. Section IV proposes a new architecture for the application using LoRa based sensor nodes and using fog computing in the current architecture along with the algorithm for fault localization. Section V shows the results of power consumption and range using LoRa and advantages for using this architecture instead of the current implementations. Main contributions of this paper are: 
- LoRa based FPIs and their assessment for range and power consumption

- Hierarchical structure for fault localization using fog computing

\section{EXISTING MODEL}

The existing implementation uses a data concentrator for sending the data to the SCADA system. The block diagram of the system is shown in Figure 1.

The fault indicators use RF/ZigBee to transmit the data from transmission lines to the data concentrator. Each phase line has one FPI installed for monitoring the values for that phase. At every intersection, there are close to nine fault indicators as shown in the Figure 1. For every intersection, there is one data concentrator and it is interfaced with the SCADA using wired (Ethernet) or wireless (GSM/GPRS) communication.

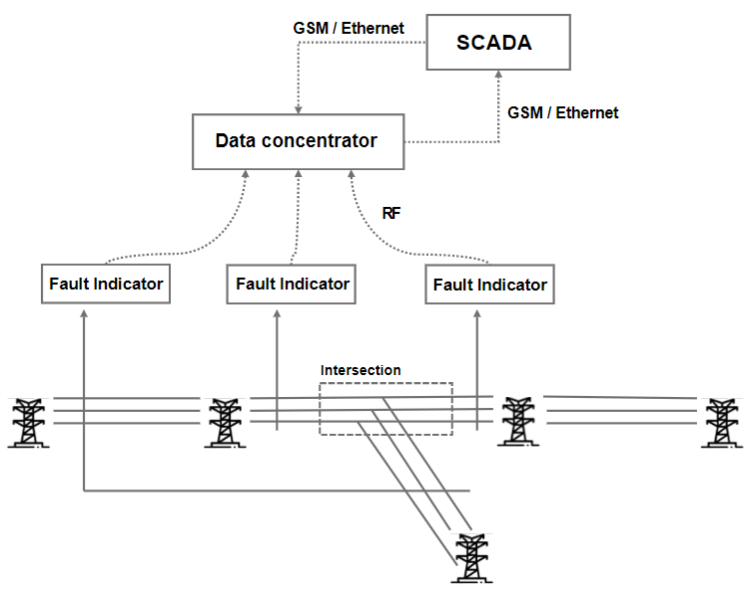

Figure 1 Existing Model

A cloud based system is not used in this scenario. Protocols like IEC103/IEC104 are used for data acquisition from the fault indicators. The advantage here is that the system is isolated and hence is more secure. When GSM/GPRS is used, the data transmission rate is dependent on the availability of the network and its speed. Usually, the data is queried from the SCADA and data concentrator responds with the instantaneous values it possesses from the FPIs. In this architecture, the electricity utility company has the mapping of the location and the data concentrator ID installed. When a fault occurs in the transmission line, the data concentrator notifies the SCADA that fault has occurred. Now, the company has information of the mapping at the location and with human intervention, the fault location is identified and fault is rectified. This is a very simple implementation and it depends on proper mapping done by humans for the installation. Although simple in execution, this is an unreliable approach due to skilled human intervention. Advanced techniques are used to remove the human intervention in any case of grid fault localization.

Another architecture is the Geographical Information System (GIS). This system is smart and works on a similar architecture. Here, advanced algorithms are programmed in the SCADA which has maps of all the sensor nodes and their values. When the values reach the SCADA system, it takes the decision to localize, isolate, and then works on the system restoration. This technique is called as FLISR (Fault Localization, Isolation, and System Restoration) technique. FLISR is an active area of research for smart grids where multiple sources of electricity are present [9], [10], [11]. In the GIS implementation, the FLISR is implemented in the SCADA system. In the proposed architecture, fault localization technique is stressed upon because the grid is not fully compatible (in Indian scenario) for automatic isolation of fault and system restoration using remote commands from the cloud. The first step in FLISR is also fault localization that is explained in detail in Figure 2 below.

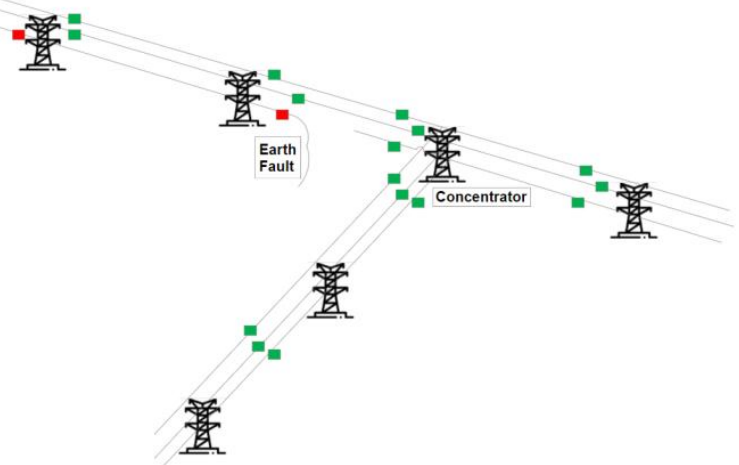

Figure 2 Fault Indication during earth fault

Figure 2 shows that when an earth fault occurs, how the fault indicators in the whole network will behave. For one earth fault, multiple data concentrators will show fault indication. The fault is detected by every FPI which comes between the generation to the fault location. This information is then relayed by the data concentrator to the GIS. The GIS computes where exactly the fault has occurred and provides the geographical location. Although, it is easier to take decision at a macro level, it involves high amounts of data computing and complex fault-finding algorithms. Then it will provide isolation to the area using circuit breakers and using remote commands. Although efficient with advanced algorithms, this system has its disadvantages which are discussed below.

- It needs complex algorithms implemented in the SCADA system and hence the algorithms are executed on all the data which might not be necessary as the fault is at a small area.

- This implementation is not cost effective. Due to less range, one data concentrator is used for each intersection node. This data concentrator then communicates to the main SCADA, so data concentrator needs to have enough data computing, storage, and battery resources.

- Consumes more power due to high data transmission rates which can affect battery life.

\section{FOG COMPUTING AND LoRa}

Fog computing is a hierarchical approach to cloud computing where the intermediate layer will do some primitive computations or segregate the data before it is sent to the cloud. The cloud will use this data for storage or further computations to find trends [12]. An intermediate layer is called as a fog node. The advantage of having a fog node is that it will reduce the access time for fault localization if the decision is taken by the cloud.

LoRa (Long Range) technology offers communication over long ranges with low power consumption. It uses the unlicensed Industrial, Scientific, and Medical (ISM) band. LoRaWAN (Long Range Wide Area Network) supported by the LoRa Alliance works on various bands available for M2M (machine-to-machine)/ (Internet of Things) IoT applications 
and uses robust Chirp Spread Spectrum modulation. LoRa shows resistance against doppler effect and multipath fading [13] that increases the range.

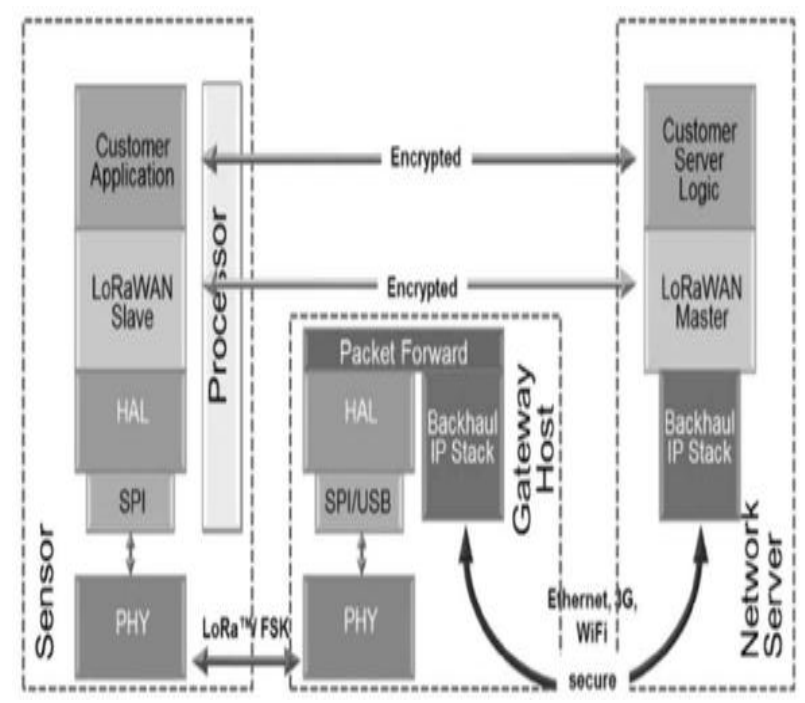

Figure 3 LoRa network block diagram

LoRa uses Coding Rate for protection of the signal against interference during the transmission but higher values of coding rate can increase the message delivery time. Each LoRa module is identified using IEEE DevEUI - 64-bit number which is unique for each node. The server also has a unique ID called IEEE AppEUI which is also 64-bit. Also, communication needs a 128-bit encryption key that is randomly generated for security purposes. The server and client should be able to encode and decode the messages using this random key. A network diagram of LoRa Wide Area Network (LoRaWAN) is shown in Figure 3. LoRa Network Block Diagram Here, a gateway host may or may not be used to transmit data to cloud. A gateway host is a service provider whose network can be used for range enhancement.

\section{LoRa BASED FAULT INDICATOR}

The FPIs used RF/ZigBee interfaced over serial UART in earlier implementations. In this research, a LoRa module from SemTech with Renesas RL78 series microcontroller is interfaced. It communicates the packets to the LoRa module and then the module transmits or receives the data. The FPIs are directly connected to the Low Power Wide Area Network (LPWAN) network and all the computing is done on the cloud. When a fault is generated in the transmission lines, all the FPIs in the line will detect the fault as shown in Figure 2. So, all the indicators will transmit the fault signal and the cloud computes fault signals from each indicator to find the fault location.

Here, each fault indicator will be programmed with the GPS coordinates of the installation site. In [14], temporary fault indicators are programmed with GPS coordinates. But, the system is very slow and needs human intervention. The GPS will help in finding the faults for the fog node as well as the cloud if the fault indicators are directly interfaced with the cloud. These coordinates will directly be programmed into the fault indicators at the time of installation. Figure 4 below shows the fault indicator interfaced with a LoRa module.

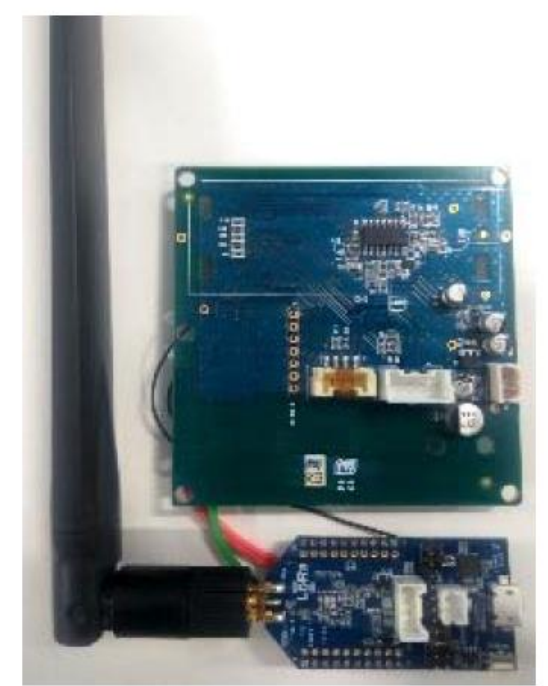

Figure 4 Fault Indicator with LoRa module

In previous sections, cloud based solution is discussed for fault localization. Here, a new architecture is proposed that implements fog computing as shown in

Figure 5. It shows an intermediate layer of fog node that will take care of the computations for fault localization. All the fault indicators are connected to this fog node. The fog node will be catering to a greater number of FPI (sensor nodes) as compared to the earlier design due to increased range of LoRa. The data from all the FPI will be processed here for faults and monitor normal operation. As the SCADA is replaced by cloud in this architecture, the data can be monitored from anywhere and will not be localized to the place of SCADA only.

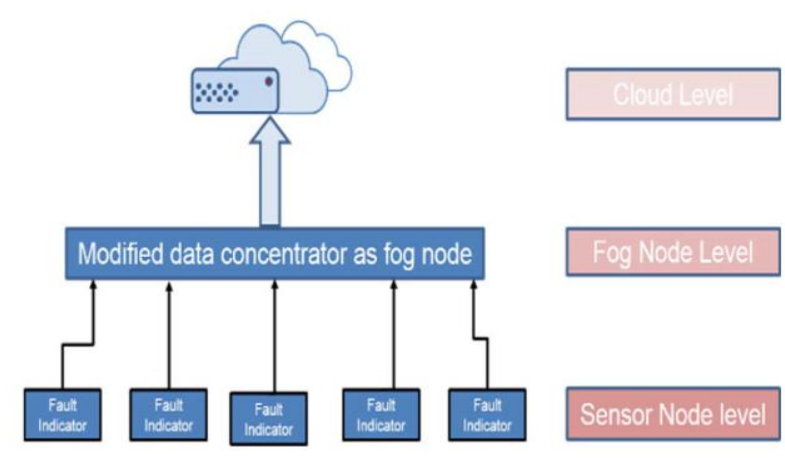

Figure 5 Fog architecture

The fog node does the following functionalities:

- Receive data from all FPI nodes and check for fault

- Find the fault location and convey it to the cloud.

- Send the data that is necessary for logging to the cloud

- Realize trends of phase fluctuations

In this architecture, each FPI node is paired with one other FPI node. Pairing two nodes at each ends of the transmission lines will reduce the transmissions drastically. When the node is being installed, it will be configured with another node and these two nodes will be paired to monitor one given length of cable. Cables can be monitored easily using paired FPI nodes. Now, when a fault occurs in the system, many faults will be 
generated as shown in Figure 2. There are two scenarios for the fault indicator of normal operation and fault operation described below.

Normal Operation: During the normal operation, the health packets must be transmitted by the FPIs. The flowchart is shown in the Figure 6 ,

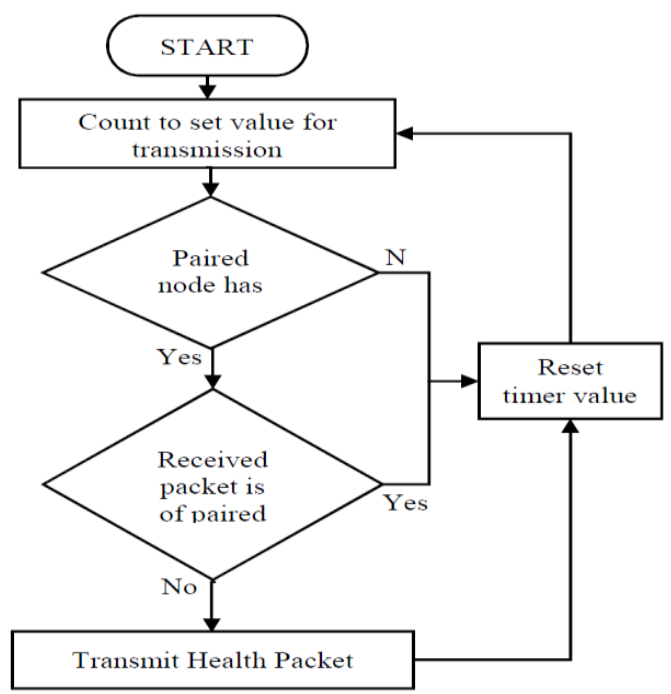

Figure 6 Normal operation flow chart

There are two paired nodes, node A and node B. Node A transmits a health packet after its designated time to the fog node. Node B and the fog node receives it. No fault in node B either, so node B does not transmit the health packet because the values will not be very different as the fault indicators are in vicinity and same phase. When the health packet timeout occurs for node B and node A has not transmitted, then Node B transmits the health packet. If one of the nodes fails to transmit, then other node will keep on transmitting health packet and notify the fog node about its failed paired node after a specified period. When a new pairing must be initiated, a pairing request to the pre-installed node must be sent and the pairing can be done. The advantage here is the number of health packet transmissions are reduced to half directly. This will reduce the power consumption for each FPI node.

Fault Operation: During fault operation, the FPIs transmit the fault to the fog node. In the fault packet, the information of the fault and location of the fault is provided to the fog node. When fault occurs, one pair of nodes has fault and fault is not present at the other end as seen in Figure 2. The 'no fault' node will transmit a 'distress signal' when it detects that its paired node has detected some fault.

When the fog node receives the faults from various FPIs, it will work on the following steps for fault localization. The steps are listed below,

- Check the number of faults.

- Store the faults and type of faults from all the nodes

- Check which node's paired node has transmitted a 'distress signal' and then compute the GPS parameters for the distance between the two nodes

- Compute the distance between the nodes and predict that the fault has occurred between these two fault indicators.

- Convey the information to the cloud for further

\section{processing}

The issue with this implementation is that if the 'distress signal' node is not present or its battery has died, then this algorithm will not work. That is why in this implementation, all the faults must be transmitted to the fog node. So, if there is no 'distress signal', even then, the localization of the fault can happen. When there is no 'distress signal' at that time the fog node will check if two continuous nodes have transmitted a fault. The fog node can then traverse through all the nodes which are in vicinity and check if they have fault. Then at the end node, the GPS locations can be found out. If the fault is out of range of the fog node, then it will know how many possible combinations are present where the fault can be found. The fog node will then determine if the fault is in its range or not. The fog node can also process all of data and send a consolidated data to the cloud for logging. This design approach provides a robust fault localization scheme.

In this research, a practical implementation of fog computing is explored for fault localization in transmission lines. Current industry trend uses a Geographical Information System (GIS) and complex algorithms to zero down on the issue in the transmission lines. Here, a simple mechanism of using GPS coordinates and pairing of two nodes must be done to get better fault localization. Initial installation will be time consuming, but this algorithm offers faster response and low operational costs.

\section{POWER CONSUMPTION}

The power consumption tables of LoRa modules at different powers are shown in Figure 7. The current consumption of ZigBee and RF is lesser than $30 \mathrm{~mA}$ while in active state and will increase when it is transmitting the packet. Also, the range covered by ZigBee/RF is very less compared to LoRa. This architecture for reduced number of transmissions will help in coping with the higher power consumption along with benefits of higher range. Figure 7 shows the consumption increase of the LoRa module with increase in the transmission power as compared to RF and ZigBee modules.

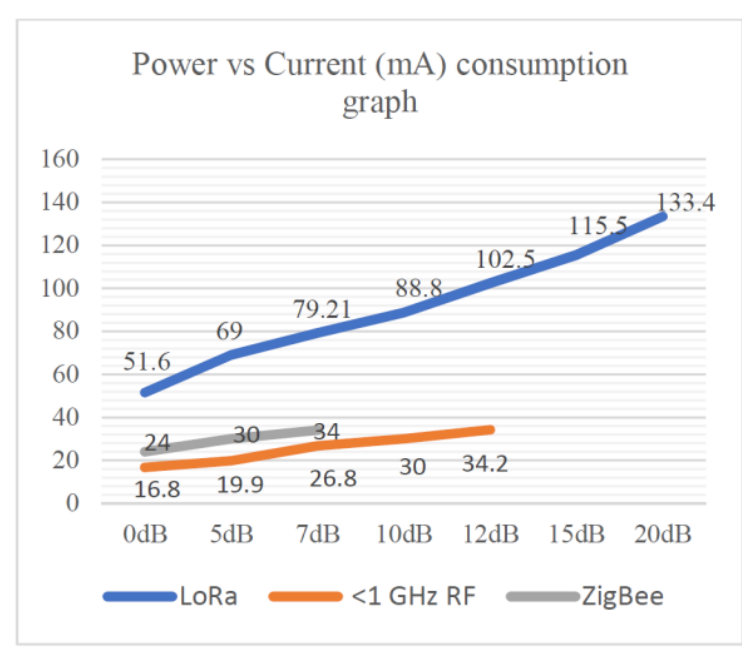

Figure 7 Power vs Current (mA) consumption graph

\section{CONCLUSION}

In this research, the future work will include the work of FPIs and the fault localization using this architecture in smart grids. Work on simulation of this system in smart grids and increase the number of paired nodes to assess the algorithm will be done. As smart grids have different sources of generation, it will be challenging to find the faults in smart grids and micro 
grids. Due to different sources, there might be more phase faults in smart grids. Also, when the fault is out of range, the fault detection or communication with another fog node can be a challenging task to further this research.

Increased range is more beneficial when LoRa based fault indicators are realized. Architecture proposed in this research has increased the range along with a minimum increase in the power consumption. An algorithm to cater the increase in the power consumption is proposed using paired nodes. In this paper, a novel approach to the fault localization has been proposed using paired nodes and GPS coordinates. A fog computing based solution will reduce the dependence of a cloud and GIS which can be costly in terms of implementation. For a better coverage, LoRa based WSN has been proposed that can cover a larger area.

\section{ACKNOWLEDGMENTS}

Our thanks to the Mr. Sushrut Lagwankar and Mr. Pratik Pimparkar how help a lot during this research and experiments.

\section{REFERENCES}

[1] L.L.Dhirani, T. Newe, E.Lewis and S.Nizamani, "Cloud computing and Internet of Things fusion: Cost issues," in 2017 Eleventh International Conference on Sensing Technology (ICST), Sydney, NSW, Australia, 4-6 Dec. 2017.

[2] M.Bouchaala, C.Ghazel, L.A.Saidane and F.Kamoun, "End to End Cloud Computing Architecture Based on A Novel Classification of Security Issues," in 14th International Conference on Computer Systems and Applications (AICCSA), Hammamet, Tunisia, 30 Oct.-3 Nov. 2017.

[3] K.Gandhi and P.Gandhi, "Cloud computing security issues: An analysis," in 3rd International Conference on Computing for Sustainable Global Development, New Delhi, India, 16-18 March 2016.

[4] J.Zhou, T.Wang and M.Z.A.Bhuiyan, "A Hierarchic Secure Cloud Storage Scheme Based on Fog Computing," in 15th Intl Dependable, Autonomic and Secure Computing,15th Intl Conf on Pervasive Intelligence \& Computing, 3rd Intl Conf on Big Data Intelligence and Computing and Cyber Science and Technology

Congress(DASC/PiCom/DataCom/CyberSciTech), Orlando, FL, USA, 6-10 Nov. 2017.

[5] S.Alharbi, P.Rodriguez, M.Rajaputhri, P.Iyer, N.Bose and Z.Ye, "FOCUS: A fog computing-based security system for the Internet of Things," in 2018 15th IEEE Annual Consumer Communications \& Networking Conference (CCNC), Las Vegas, NV, USA., 12-15 Jan. 2018.
[6] R.Brzoza-Woch, M.Konieczny, P.Nawrocki, T.Szydlo and K.Zielinski, "Embedded systems in the application of fog computing — Levee monitoring use case," in 2016 11th IEEE Symposium on Industrial Embedded Systems (SIES), Krakow, Poland, 23-25 May 2016.

[7] H.O.Cruz and F.B.Leão, "Optimal placement of fault indicators using adaptive genetic algorithm," in 2017 IEEE Power \& Energy Society General Meeting, Chicago, IL, USA, 16-20 July 2017.

[8] Z.Yang, W.Wei, S.Ziang, F.Kai-Jun and X.Bing-Yin, "Networking technology of fault indication system based on ZigBee," in PES General Meeting | Conference \& Exposition, 2014 IEEE, National Harbor, MD, USA, $27-$ 31 July 2014

[9] R.A.Spalding, L.H.L.Rosa, C.F.M. Almeida, R.F.Morais, M.R.Gouvea, N.Kagan, D.Mollica, A.Dominice, L.Zamboni, G.h.Batista, J.P.Silva, L.A.Costa and M.A.Fredes, "Fault Location, Isolation and service restoration (FLISR) functionalities tests in a Smart Grids laboratory for evaluation of the quality of service," in 17th International Conference on Harmonics and Quality of Power (ICHQP), Belo Horizonte, Brazil, 16-19 Oct. 2016.

[10] P.Jamborsalamati, A.Sadu, F.Ponci and A.Monti, "Design, implementation and real-time testing of an IEC 61850 based FLISR algorithm for smart distribution grids," in IEEE International Workshop on Applied Measurements for Power Systems (AMPS), Aachen, Germany, 23-25 Sept. 2015.

[11] A.Shahsavari, A.Fereidunian, A.Ameli, S.M.Mazhari and H.Lesani, "A healer reinforcement approach to smart grids by improving fault location function in FLISR," in 13th International Conference on Environment and Electrical Engineering, Wroclaw, Poland, 1-3 Nov. 2013.

[12] Y.Liu, J.E.Fieldsend and G.Min, "A Framework of Fog Computing: Architecture, Challenges, and Optimization," Cyber-Physical-Social Computing and Networking, vol. 5, pp. 25445 - 25454, 26 October 2017.

[13] A.Javed, H.Larijani, A.Wixted and R.Emmanuel, "Random Neural Networks based Cognitive Controller for HVAC in Non-Domestic Building using LoRa," in 16th International Conference on Cognitive Informatics \& Cognitive Computing (ICCI*CC), Oxford, UK, 26-28 July 2017

[14] R.M.Capelini, G.M.F.Ferraz, R.Salustiano, E.T.W.Neto, M.L.Pereira, Â.R.Oliveira and R.D.Testi, "Methodology for fast fault location in overhead distribution networks by the application of temporary georeferenced fault indicators," in International Conference on High Voltage Engineering and Application (ICHVE), Chengdu, China, 19-22 Sept. 2016 